\title{
List Edge and List Total Colorings of Planar Graphs without non-induced 7-cycles $\|^{\dagger}$
}

\author{
Aijun Dong 非 \\ Guizhen Liu非 \\ Guojun $\mathrm{Li}^{2} \mid$ \\ ${ }^{1}$ School of science, Shandong Jiaotong University, Jinan 250023, P. R. China \\ ${ }^{2}$ School of Mathematics, Shandong University, Jinan 250100, P. R. China \\ received $22^{\text {nd }}$ November 2010, revised $11^{\text {th }}$ October 2011, 10 $0^{\text {th }}$ July 2012, accepted $19^{\text {th }}$ February 2013.
}

Giving a planar graph $G$, let $\chi_{l}^{\prime}(G)$ and $\chi_{l}^{\prime \prime}(G)$ denote the list edge chromatic number and list total chromatic number of $G$ respectively. It is proved that if $G$ is a planar graph without non-induced 7-cycles, then $\chi_{l}^{\prime}(G) \leq \Delta(G)+1$ and $\chi_{l}^{\prime \prime}(G) \leq \Delta(G)+2$ where $\Delta(G) \geq 7$.

Keywords: List coloring; Planar graph; Choosability.

\section{Introduction}

The terminology and notation used but undefined in this paper can be found in [1]. Let $G$ be a graph and we use $V(G), E(G), F(G), \Delta(G)$ and $\delta(G)$ to denote the vertex set, edge set, face set, maximum degree, and minimum degree of $G$, respectively. Let $d_{G}(x)$ or simply $d(x)$, denote the degree of a vertex (resp. face) $x$ in $G$. A vertex (resp. face) $x$ is called a $k$-vertex (resp. $k$-face), $k^{+}$-vertex (resp. $k^{+}$-face), or $k^{-}$-vertex, if $d(x)=k, d(x) \geq k$, or $d(x) \leq k$. We use $\left(d_{1}, d_{2}, \cdots, d_{n}\right)$ to denote a face $f$ if $d_{1}, d_{2}, \cdots, d_{n}$ are the degrees of vertices which are incident with the face $f$. If $u_{1}, u_{2}, \cdots, u_{n}$ are the vertices on the boundary walk of a face $f$, then we write $f=u_{1} u_{2} \cdots u_{n}$. Let $\delta(f)$ denote the minimal degree of vertices which are incident with $f$. We use $f_{i}(v)$ to denote the number of $i$-faces which are incident with $v$ for each $v \in V(G)$. Let $n_{i}(f)$ denote the number of $i$-vertices which are incident with $f$ for each $f \in F(G)$. A cycle $C$ of length $k$ is called $k$-cycle, and if there is at least one edge $x y \in E(G) \backslash E(C)$ and $x, y \in V(C)$, the cycle $C$ is called non-induced $k$-cycle.

The mapping $L$ is said to be a total assignment for a graph $G$ if it assigns a list $L(x)$ of possible colors to each element $x \in V(G) \cup E(G)$. If $G$ has a proper total coloring $\phi(x) \in L(x)$ for all $x \in V(G) \cup E(G)$, then we say that $G$ is total-L-colorable. Let $f: V(G) \cup E(G) \rightarrow N$ where $f$ is a function into the positive integers. We say that $G$ is total-f-choosability if it is total- $L$-colorable for every total assignment $L$

\footnotetext{
${ }^{\dagger}$ Supported by NSFC grants $11101243,11161035$.

‡E-mail: dongaijun@mail.sdu.edu.cn

EE-mail: gzliu@sdu.edu.cn.

ฯE-mail: guojunsdu@ gmail.com.

1365-8050 @ 2013 Discrete Mathematics and Theoretical Computer Science (DMTCS), Nancy, France
} 
satisfying $|L(x)|=f(x)$ for all $x \in V(G) \cup E(G)$. The list total coloring number $\chi_{l}^{\prime \prime}(G)$ of $G$ is the smallest integer $k$ such that $G$ is total- $f$-choosability when $f(x)=k$ for each $x \in V(G) \cup E(G)$. The list edge coloring number $\chi_{l}^{\prime}(G)$ of $G$ is defined similarly in terms of coloring edges alone; and so is the concept of edge-f-choosability. On the list coloring number of a graph $G$, there is a famous conjecture known as the List Coloring Conjecture.

Conjecture 1 For a multigraph $G$,

$$
\text { (a) } \chi_{l}^{\prime}(G)=\chi^{\prime}(G) ; \quad(b) \chi_{l}^{\prime \prime}(G)=\chi^{\prime \prime}(G) .
$$

Part $(a)$ of Conjecture 1 was formulated independently by Vizing, by Gupta, by Alberson and Collins, and by Bollobás and Harris [6, 11]. It is well known as the List Coloring Conjecture. Part (b) was formulated by Borodin, Kostochka and Woodall [2]. Part $(a)$ has been proved for bipartite multigraphs [5]. Part $(a)$ and Part $(b)$ have been proved for outerplanar graphs [15], and graphs with $\Delta \geq 12$ which can be embedded in a surface of nonnegative characteristic [2]. There are several related results for planar graphs, such as planar graphs without 4-cycles by Hou et al.[9], planar graphs without 4- and 5-cycles or planar graphs without intersecting 4-cycles by Liu et al.[13], planar graphs without triangles adjacent 4-cycles by Li et al.[14], planar graphs without intersecting triangles by Sheng et al.[18].

To confirm Conjecture 1 is a challenging work. From the Vizing Theorem and the Total Coloring Conjecture, the following weak conjecture is presented.

Conjecture 2 For a multigraph $G$,

$$
\text { (a) } \chi_{l}^{\prime}(G) \leq \Delta(G)+1 ; \quad(b) \chi_{l}^{\prime \prime}(G) \leq \Delta(G)+2 .
$$

Part $(a)$ of Conjecture 2 has been proved for complete graphs of odd order [7]. Wang et al. confirmed part $(a)$ of Conjecture 2 for planar graphs without 6 -cycles or without 5 -cycles [17, 16]. Zhang et al. proved part $(a)$ of Conjecture 2 for planar graphs without triangles [19]. Hou et al. proved part $(a)$ of Conjecture 2 for planar graphs without adjacent triangles or 7-cycles [8]. Cai et al. confirmed part $(a)$ of Conjecture 2 for planar graphs without chordal 5 -cycles [3]. Part $(b)$ of Conjecture 2 was proved by Hou et al. for planar graphs $G$ with $\Delta(G) \geq 9$ [10]. Dong et al. confirmed Conjecture 2 for planar graphs without 6 -cycles with chord [4].

In this paper, we shall show the following result.

Theorem Let $G$ be a planar graph without non-induced 7-cycles, if $\Delta(G) \geq 7$, then $\chi_{l}^{\prime}(G) \leq \Delta(G)+1$ and $\chi_{l}^{\prime \prime}(G) \leq \Delta(G)+2$.

\section{Planar graphs without non-induced 7-cycles}

First let us introduce some important lemmas.

Lemma 3 Let $G$ be a planar graph without non-induced 7-cycles. Then there is an edge uv $\in E(G)$ such that $\min \{d(u), d(v)\} \leq\left\lfloor\frac{\Delta(G)+1}{2}\right\rfloor$ and $d(u)+d(v) \leq \max \{9, \Delta(G)+2\}$.

Proof: Suppose to the contrary that $G$ is a minimal counterexample to Lemma 3 in terms of the number of vertices and edges. Then we have $\delta(G) \geq 3$. 
By Euler's formula $|V|-|E|+|F|=2$ and $\sum_{v \in V(G)} d(v)=\sum_{f \in F(G)} d(f)=2|E|$, we have

$$
\sum_{v \in V(G)}(2 d(v)-6)+\sum_{f \in F(G)}(d(f)-6)=-6(|V|-|E|+|F|)=-12 .
$$

Define an initial charge function $w$ on $V(G) \cup F(G)$ by setting $w(v)=2 d(v)-6$ if $v \in V(G)$ and $w(f)=d(f)-6$ if $f \in F(G)$, so that $\sum_{x \in V(G) \cup F(G)} w(x)=-12$. Now redistribute the charge according to the following discharging rules.

For convenience, let $\bar{w}(v)$ denote the total charge transferred from a vertex $v$ to all its incident 4- and 5 -faces where $d(v)=5$.

$D 1$ Let $f$ be a 3-face incident with a vertex $v$. Then $v$ gives $f$ charge $\frac{4-\bar{w}(v)}{f_{3}(v)}$ if $d(v)=5, \frac{3}{2}$ if $d(v) \geq 6$.

$D 2$ Let $f$ be a 4-face incident with a vertex $v$. Then $v$ gives $f$ charge $\frac{1}{2}$ if $d(v)=4,5$ and 6,1 if $d(v) \geq 7$.

$D 3$ Let $f$ be a 5-face incident with a vertex $v$. Then $v$ gives $f$ charge $\frac{1}{5}$ if $d(v)=4,5$ and $6, \frac{1}{3}$ if $d(v) \geq 7$.

Let the new charge of each element $x$ be $w^{\prime}(x)$ for each $x \in V(G) \cup F(G)$.

In the following, let us check the new charge $w^{\prime}(x)$ of each element $x \in V(G) \cup F(G)$.

Suppose $d(v)=3$. Then $w^{\prime}(v)=w(v)=0$.

Suppose $d(v)=4$. Then $w(v)=2, f_{4}(v) \leq 4$. If $2 \leq f_{4}(v) \leq 4$, then $f_{5}(v)=0$ for $G$ contains no non-induced 7-cycles. We have $w^{\prime}(v) \geq 2-\frac{1}{2} \times 4=0$ by $D 2$. Otherwise, i.e. $f_{4}(v) \leq 1$, then $f_{5}(v) \leq 4$. Thus we have $w^{\prime}(v)>2-\frac{1}{2}-\frac{1}{5} \times 4=\frac{7}{10}>0$ by $D 2$ and $D 3$.

Suppose $d(v)=5$. Then $w(v)=4, f_{3}(v) \leq 5$. If $1 \leq f_{3}(v)$, then $w^{\prime}(v) \geq 4-\frac{4-\bar{w}(v)}{f_{3}(v)} f_{3}(v)-\bar{w}(v)=0$ by $D 1$. Otherwise, i.e. $f_{3}(v)=0$, then $f_{4}(v)+f_{5}(v) \leq 5$. It is clear that $w^{\prime}(v)>4-\frac{1}{2} \times 5=\frac{3}{2}>0$ by $D 2$ and $D 3$.

Suppose $d(v)=6$. Then $w(v)=6, f_{3}(v) \leq 4$ for $G$ contains no non-induced 7-cycles. If $f_{3}(v)=4$, then $f_{4}(v)=0$ and $f_{5}(v)=0$ for $G$ contains no non-induced 7 -cycles. We have $w^{\prime}(v) \geq 6-\frac{3}{2} \times 4=0$ by $D 1$. If $f_{3}(v) \leq 3$, then it is clear that $w^{\prime}(v)>6-\frac{3}{2} \times 3-\frac{1}{2} \times 3=0$ by $D 1, D 2$ and $D 3$.

Suppose $d(v)=7$. Then $w(v)=8, f_{3}(v) \leq 5$ for $G$ contains no non-induced 7 -cycles.

Suppose $f_{3}(v)=5$. Then $f_{4}(v)=0$ and $f_{5}(v)=0$ for $G$ contains no non-induced 7 -cycles. We can get $w^{\prime}(v) \geq 8-\frac{3}{2} \times 5=\frac{1}{2}>0$ by $D 1$.

Suppose $f_{3}(v)=4$. Then $f_{4}(v) \leq 2$. If $f_{4}(v)=2$, then $f_{5}(v)=0$ for $G$ contains no non-induced 7-cycles. We have $w^{\prime}(v) \geq 8-\frac{3}{2} \times 4-1 \times 2=0$ by $D 1$ and $D 2$. If $f_{4}(v) \leq 1$, then $f_{5}(v) \leq 1$ for $G$ contains no non-induced 7-cycles. We have $w^{\prime}(v) \geq 8-\frac{3}{2} \times 4-1-\frac{1}{3}=\frac{2}{3}>0$ by D1, D2 and D3.

Suppose $f_{3}(v)=3$. Then $f_{4}(v) \leq 2$ and $f_{5}(v) \leq 2$ for $G$ contains no non-induced 7 -cycles. It is clear that $w^{\prime}(v)>8-\frac{3}{2} \times 3-1 \times 2-\frac{1}{3} \times 2=\frac{5}{6}>0$ by $D 1, D 2$ and $D 3$.

Suppose $f_{3}(v) \leq 2$. Then it is clear that $w^{\prime}(v)>8-\frac{3}{2} \times 2-1 \times 5=0$ by $D 1, D 2$ and $D 3$.

Suppose $d(v)=8$. Then $w(v)=10, f_{3}(v) \leq 6$ for $G$ contains no non-induced 7 -cycles. If $f_{3}(v)=6$, then $f_{4}(v)=0$ and $f_{5}(v)=0$ for $G$ contains no non-induced 7 -cycles. We have $w^{\prime}(v) \geq 10-\frac{3}{2} \times 6=$ $1>0$ by $D 1$. If $f_{3}(v)=5$, then $f_{4}(v) \leq 1$ and $f_{5}(v) \leq 1$ for $G$ contains no non-induced 7 -cycles. We can get $w^{\prime}(v) \geq 10-\frac{3}{2} \times 5-1-\frac{1}{3}=\frac{7}{6}>0$ by $D 1, D 2$ and $D 3$. If $f_{3}(v) \leq 4$, then it is clear that $w^{\prime}(v) \geq 10-\frac{3}{2} \times 4-1 \times 4=0$ by $D 1, D 2$ and $D 3$. 
Suppose $d(v)=9$. Then $w(v)=12, f_{3}(v) \leq 7$ for $G$ contains no non-induced 7-cycles. If $f_{3}(v)=7$, then $f_{4}(v)=0$ and $f_{5}(v)=0$ for $G$ contains no non-induced 7 -cycles. We can get $w^{\prime}(v) \geq 12-\frac{3}{2} \times 7=$ $\frac{3}{2}>0$ by $D 1$. If $f_{3}(v) \leq 6$, then it is clear that $w^{\prime}(v)>12-\frac{3}{2} \times 6-1 \times 3=0$ by $D 1, D 2$ and $D 3$.

Suppose $d(v) \geq 10$. Then $w(v)=2 d(v)-6, f_{4}(v)+f_{5}(v) \leq d(v)-f_{3}(v)$. Thus we have $w^{\prime}(v) \geq$ $2 d(v)-6-\frac{3}{2} f_{3}(v)-f_{4}(v)-\frac{1}{3} f_{5}(v) \geq d(v)-6-\frac{1}{2} f_{3}(v)$ by $D 1, D 2$ and $D 3$. Since $f_{3}(v) \leq \frac{4}{5} d(v)$, we have $w^{\prime}(v) \geq \frac{3}{5} d(v)-6 \geq 0$.

Suppose $d(f)=3$. Then $w(f)=-3$.

Suppose $\delta(f)=3$. Then $f$ is a $\left(3,7^{+}, 7^{+}\right)$-face by assumption. We have $w^{\prime}(f)=-3+\frac{3}{2} \times 2=0$ by $D 1$. $D 1$.

Suppose $\delta(f)=4$. Then $f$ is a $\left(4,6^{+}, 6^{+}\right)$-face by assumption. We have $w^{\prime}(f)=-3+\frac{3}{2} \times 2=0$ by

Suppose $\delta(f)=5$. Then $f$ is a $\left(5,5^{+}, 5^{+}\right)$-face.

Suppose $f$ is a $(5,5,5)$-face. For convenience, let $f=u v w$. Of the three vertices $u, v$ and $w$, there is at most one vertex which is incident with at least four 3 -faces for the reason that $G$ contains no non-induced 7 -cycles. Without loss of generality, let $f_{3}(u) \geq 4$. Then $f_{3}(u)+f_{4}(u)+f_{5}(u) \leq 5, f_{3}(v)+f_{4}(v)+$ $f_{5}(v) \leq 3$ and $f_{3}(w)+f_{4}(w)+f_{5}(w) \leq 3$ for $G$ contains no non-induced 7 -cycles. We have $\frac{4-\bar{w}(v)}{f_{3}(u)} \geq \frac{4}{5}$, $\frac{4-\bar{w}(v)}{f_{3}(v)} \geq \frac{4}{3}, \frac{4-\bar{w}(v)}{f_{3}(w)} \geq \frac{4}{3}$ by $D 2$ and $D 3$. Thus $w^{\prime}(f) \geq-3+\frac{4}{5}+\frac{4}{3} \times 2=\frac{7}{15}>0$ by $D 1$. Now we assume that $f_{3}(u) \leq 3, f_{3}(v) \leq 3, f_{3}(w) \leq 3$. Then we have $\frac{4-\bar{w}(v)}{f_{3}(v)} \geq 1$ for $G$ contains no non-induced 7-cycles and by $D 1, D 2$ and $D 3$. Thus $w^{\prime}(f) \geq-3+1 \times 3=0$ by $D 1$.

Suppose $f$ is a $\left(5,5,6^{+}\right)$-face. For convenience, let $f=u v w$ where $d(u)=d(v)=5$. Since $f_{3}(u)+f_{4}(u)+f_{5}(u) \leq 5, f_{3}(v)+f_{4}(v)+f_{5}(v) \leq 5$, we have $\frac{4-\bar{w}(v)}{f_{3}(u)} \geq \frac{4}{5}, \frac{4-\bar{w}(v)}{f_{3}(v)} \geq \frac{4}{5}$ by $D 2$ and D3. Thus $w^{\prime}(f) \geq-3+\frac{4}{5} \times 2+\frac{3}{2}=\frac{1}{10}>0$ by $D 1$.

Suppose $f$ is a $\left(5,6^{+}, 6^{+}\right)$-face. Then we have $w^{\prime}(f)>-3+\frac{3}{2} \times 2=0$ by $D 1$.

Suppose $\delta(f) \geq 6$. Then we have $w^{\prime}(f)=-3+\frac{3}{2} \times 3=\frac{3}{2}>0$ by $D 1$.

Suppose $d(f)=4$. Then $w(f)=-2$. If $\delta(f)=3$, then $f$ is a $\left(3,3^{+}, 7^{+}, 7^{+}\right)$-face by assumption. We have $w^{\prime}(f) \geq-2+1 \times 2=0$ by $D 2$. If $\delta(f) \geq 4$, then $f$ is a $\left(4^{+}, 4^{+}, 4^{+}, 4^{+}\right)$-face. We have $w^{\prime}(f) \geq-2+\frac{1}{2} \times 4=0$ by $D 2$.

Suppose $d(f)=5$. Then $w(f)=-1$.

Suppose $\delta(f)=3$. Then $n_{3}(f) \leq 2$ by assumption. If $n_{3}(f)=2$, then $f$ is a $\left(3,3,7^{+}, 7^{+}, 7^{+}\right)$-face by assumption. We have $w^{\prime}(f) \geq-1+\frac{1}{3} \times 3=0$ by $D 3$. If $n_{3}(f)=1$, then $f$ is a $\left(3,4^{+}, 4^{+}, 7^{+}, 7^{+}\right)$-face by assumption. We have $w^{\prime}(f) \geq-1+\frac{1}{3} \times 2+\frac{1}{5} \times 2=\frac{1}{15}>0$ by $D 3$.

Suppose $\delta(f) \geq 4$. Then we have $w^{\prime}(f) \geq-1+\frac{1}{5} \times 5=0$ by $D 3$.

Suppose $d(f) \geq 6$. Then $w^{\prime}(f)=w(f) \geq 0$.

From the above discussion, we obtain $-12=\sum_{x \in V(G) \cup F(G)} w^{\prime}(x) \geq 0$, a contradiction.

Lemma 4 Let $G$ be a planar graph without non-induced 7 -cycles. Then $\chi_{l}^{\prime}(G) \leq k+1$ and $\chi_{l}^{\prime \prime}(G) \leq$ $k+2$ where $k=\max \{\Delta(G), 7\}$.

Proof: Suppose to the contrary that $G^{\prime}$ and $G^{\prime \prime}$ are minimal counterexamples to the conclusions for $\chi_{l}^{\prime}$ and $\chi_{l}^{\prime \prime}$ respectively. Let $L^{\prime}$ and $L^{\prime \prime}$ be list assignments such that $\left|L^{\prime}(e)\right|=k+1$ for each $e \in E(G), G^{\prime}$ is not edge- $L^{\prime}$-colorable, and $\left|L^{\prime \prime}(x)\right|=k+2$ for each $x \in V(G) \cup E(G), G^{\prime \prime}$ is not total- $L^{\prime \prime}$-colorable. By Lemma 3. $G^{\prime}$ and $G^{\prime \prime}$ contain an edge $u v \in E(G)$ such that $\min \{d(u), d(v)\} \leq\left\lfloor\frac{\Delta(G)+1}{2}\right\rfloor$ and $d(u)+d(v) \leq \max \{9, \Delta(G)+2\}=k+2$. 
Let $\bar{G}^{\prime}=G^{\prime}-u v$. Then $\bar{G}^{\prime}$ is edge- $L^{\prime}$-colorable by assumption. For $d(u)+d(v) \leq k+2$, there are at most $k$ edges which are adjacent to $u v$ in $\bar{G}^{\prime}$. Thus there is at lest one color in $L^{\prime}(u v)$ which we can use to color $u v$. Then $G^{\prime}$ is edge- $L^{\prime}$-colorable, a contradiction.

Let $\bar{G}^{\prime \prime}=G^{\prime \prime}-u v$. Then $\overline{G^{\prime \prime}}$ is total- $L^{\prime \prime}$-colorable by assumption. With loss of generality, let $d(u)=$ $\min \{d(u), d(v)\}$. Erase the color on $u$, then there is at least one color in $L^{\prime \prime}(u v)$ which we can use to color $u v$ for $d(u)+d(v) \leq k+2$. For $d(u) \leq\left\lfloor\frac{\Delta(G)+1}{2}\right\rfloor \leq\left\lfloor\frac{k+1}{2}\right\rfloor$, then $u$ is adjacent to at most $\left\lfloor\frac{k+1}{2}\right\rfloor$ vertices and is incident with at most $\left\lfloor\frac{k+1}{2}\right\rfloor$ edges. Thus there is at least one color in $L^{\prime \prime}(u)$ which we can use to color $u$. Then $G^{\prime \prime}$ is total- $L^{\prime \prime}$-colorable, a contradiction. From the above discussion, we have $\chi_{l}^{\prime}(G) \leq k+1$ and $\chi_{l}^{\prime \prime}(G) \leq k+2$ where $k=\max \{\Delta(G), 7\}$.

By Lemma 4, it is easy to obtain the main theorem.

Theorem Let $G$ be a planar graph without non-induced 7-cycles, if $\Delta(G) \geq 7$, then $\chi_{l}^{\prime}(G) \leq \Delta(G)+1$ and $\chi_{l}^{\prime \prime}(G) \leq \Delta(G)+2$.

\section{References}

[1] J. A. Bondy and U. S. R. Murty, Graph Theory with Applications, North-Holland, New York, 1976.

[2] O. Borodin, A. Kostochka, D. Woodall, List edge and list total colourings of multigraphs, J. Combin. Theory Ser. B 71 (1997) 184-204.

[3] J. Cai, J. Hou, X. Zhang, G. Liu, Edge-choosability of planar graphs without non-induced 5-cycles, Information Processing Letters, 109 (7) (2009) 343-346.

[4] A. DONG, G. Liu, G. Li, List edge and list total colorings of planar graphs without 6-cycles with chord, Bull. Korean Math. Soc. 49 (2012) 359-365.

[5] F. Galvin, The list chromatic index of a bipartite multigraph, J. Combin. Theory Ser. B 63 (1995) 153-158.

[6] R. Hägkvist, A. Chetwynd, Some upper bounds on the total and list chromatic numbers of multigraphs, J. Graph Theory 16 (1992) 503-516.

[7] R. Hägkvist, J, Janssen, New bounds on the list-chromatic index of the complete graph and other simple graphs, Combin. Probab. Comput. 6 (1997) 295-313.

[8] J. Hou, G. Liu, J. Cai, Edge-choosability of planar graphs without adjacent triangles or without 7-cycle, Discrete Mathematics, 309 (2009) 77-84.

[9] J. Hou, G. Liu, J. Cai, List Edge and List Total Colorings of Planar Graphs without 4-cycles, Theoretical Computer Science, 369 (2006) 250-255.

[10] J. Hou, G. Liu, J. Wu, Some results on list total colorings of planar graphs, Lecture Note in Computer Science, 4489 (2007) 320-328.

[11] T. Jensen, B. Toft, Graph Coloring Problem, Wiley-Interscience, New York, 1995. 
[12] A. Kostochka, List edge chromatic number of graphs with large girth, Discrete Math. 101 (1992) 189-201.

[13] B. Liu, J. Hou, G. Liu, List edge and list total colorings of planar graphs without short cycles, Information Processing Letters, 108 (2008) 347-351.

[14] R. Li, B. Xu, Edge choosability and total choosability of planar graphs without no 3-cycles adjacent 4-cycles, Discrete Mathematics, 311 (2011) 2158-2163.

[15] W. Wang, K. Lih, Choosability, edge-choosability and total choosability of outerplane graphs, European J. Combin. 22 (2001) 71-78.

[16] W. Wang, K. Lih, Choosability and edge choosability of planar graphs without five cycles, Appl. Math. Lett. (2002) 15 561-565.

[17] W. Wang, K. Lih, Structure properties and edge choosability of planar graphs without 6-cycles, Combin. Probab. Comput. 10 (2001) 267-276.

[18] H. Sheng, Y. Wang, A structural theorem for planar graphs with some applications, Discrete Applied Mathematics, 159 (2011) 1183-1187.

[19] L. Zhang, B. Wu, Edge choosability of planar graphs without small cycles, Discrete Math. 283 (2004) 289-293. 